\title{
Evaluation of Automatic Blood Analyzer as Screening Method in Fetomaternal Hemorrhage
}

\author{
Marcella R. Cardoso, ${ }^{1}$ Caroline N. de Souza-Araújo, ${ }^{2}$ Maria Cecília R. Talarico, \\ Juliana Heinrich-Mouçouçah, ${ }^{2}$ Fernando Guimarães, ${ }^{2}$ and Ricardo Barini ${ }^{1}{ }^{1}$ \\ ${ }^{1}$ Department of Obstetrics and Gynecology, University of Campinas School of Medicine, Rua Alexander Fleming 101, \\ 13083-891 Campinas, Brazil \\ ${ }^{2}$ Women's Hospital Prof. Dr. José Aristodemo Pinotti, Centro de Atenção Integral à Saúde da Mulher (CAISM), \\ University of Campinas, Rua Alexander Fleming 101, 13083-891 Campinas, Brazil
}

Correspondence should be addressed to Ricardo Barini; drbarini@gmail.com

Received 27 September 2018; Revised 5 December 2018; Accepted 31 January 2019; Published 26 February 2019

Academic Editor: Jonathan Muraskas

\begin{abstract}
Copyright ( 2019 Marcella R. Cardoso et al. This is an open access article distributed under the Creative Commons Attribution License, which permits unrestricted use, distribution, and reproduction in any medium, provided the original work is properly cited.
\end{abstract}

\begin{abstract}
Screening of fetomaternal hemorrhage (FMH) is essential in management of fetomaternal antigen incompatibilities of blood. The objective in this study was to evaluate the ability of automatic blood analyzer (ABA) to screen FMH, also comparing this method with flow cytometry (FCM). The contents of fetal red blood cells and fetal hemoglobin were evaluated by FCM and ABA, respectively, using both blood samples of male adults laced with umbilical cord blood diluted at 1/10, 1/100, 1/1,000, and 1/10,000, or blood from puerperal women collected within 48 hours following delivery. FCM had better performance (area under curve, $\mathrm{AUC}=0.8723$ ) than ABA (AUC $=0.6569)$ in detecting fetal blood laced with blood from male adults. At a critical level of $0.5 \%$, ABA indicated that $27.5 \%$ of puerperal women would have FMH while FCM did not detect FMH. Our results showed that ABA overestimates FMH and disagrees with FCM on indicating puerperal women with FMH. ABA is inadequate for being used to screen for or to measure FMH.
\end{abstract}

\section{Introduction}

A correct and rapid detection and quantification of fetomaternal hemorrhage $(\mathrm{FMH})$ is essential for the management and treatment of $\mathrm{RhD}$ discordant pregnancies. The amount of fetal blood transferred into the maternal blood circulation determines the prophylactic dose of anti-D immunoglobulin to prevent isoimmunization during pregnancy and postpartum [1].

FMH is the process of transferring blood from the fetus to the maternal intravascular compartment due to chorionic villus bleeding. Isoimmunization may occur in a $\mathrm{RhD}$ negative mother carrying a $\mathrm{RhD}$ positive fetus. This may lead to the production of anti-D antibody causing the development of hemolytic disease and severe anemia of the fetus and newborns [2-4]. The purpose of prophylaxis is to prevent $\mathrm{RhD}$ isoimmunization in future pregnancies. However, prophylaxis is based on the administration of standard doses of anti-D immunoglobulin which frequently corresponds to an overtreatment of the patient. Adequate doses of immunoglobulin rely on the accurate evaluation of the amounts of fetal blood in women's circulation, using methods with limited availability [5].

The first method developed for FMH quantification is a technique based on acid elution of blood smear, known as Kleihauer-Betke test [6], which is currently obsolete [6-9]. Flow cytometry (FCM) has become the standard method for detecting and measuring FMH and is commercially available as an assay kit. FCM combines cell morphology and conjugates of fluorophore molecules with monoclonal antibodies for multiparametric analysis of specific cell population. FCM evaluation of FMH is achieved by using anti-HbF and anti$\mathrm{RhD}$ antibodies to identify a specific group of blood antigens that act as markers for red blood cells (RBC) from fetus. Anti$\mathrm{HbF}$ monoclonal antibodies allow the discrimination of three distinct cell populations: fetal RBCs, F-cells, and adult RBCs. 
FCM identification of F-cells eliminates the disadvantage of Kleihauer-Betke [10-12].

Automatic blood analyzers (ABA) combine different analytical procedures into a single device, allowing rapid determination of multiple blood parameters using a small sample volume. Automatic blood analyzer is used to define standards of accuracy, reliability, and performance of blood gas tests by measuring parameters of $\mathrm{pH}$, electrolytes, metabolites, and oximetry using spectrophotometry technology as in the case of fetal hemoglobin dosage [13]. Since it discriminates adult and fetal hemoglobin, this device is potentially useful for the evaluation of FMH. Additionally, ABA would represent a quicker and lower-cost alternative for the assessment of FMH compared to Kleihauer-Betke and/or FCM [14]. The objective in this study was to evaluate the ability of ABA to screen for FMH at critical level of $0.5 \%$ which is within the hemorrhage volumes neutralized by standard doses of anti$\mathrm{D}$ treatment, also comparing ABA with FCM, a method that has been replacing Kleihauer-Betke test for this end.

\section{Subject and Methods}

2.1. Subjects and Blood Collection. Blood samples were collected from 57 male adults weighing more than $50 \mathrm{~kg}$, who denied hematological pathologies and metabolic diseases. Blood samples from 14 newborns weighing above $2.5 \mathrm{~kg}$ were collected from the umbilical cord just after birth. Additionally, blood samples from 40 puerperal women were collected within 48 hours following delivery at Hospital da Mulher Prof. Dr. José Aristodemo Pinotti (CAISM), Women's Hospital at University of Campinas (UNICAMP, Campinas, Brazil). All blood samples of umbilical cord or puerperal women were collected from subjects who denied metabolic or hematological diseases and delivered without obstetric complications. Blood collections were performed using $10 \mathrm{ml}$ vacuum blood-sampling tubes containing sodium heparin (Vacuette, Campinas, Brazil). The study was approved by the Ethical Review Board, Research Ethics Committee of UNICAMP (1.386.253/2016), and was registered on the Brazilian National Health Council (CAAE: 49989315.0.000.5404). Subjects or their legal guardian received information about the study and gave written informed consent before blood sampling.

2.2. Blood Sample Preparation. Blood samples containing fetal blood were prepared by serially diluting blood taken from umbilical cord with blood of male adults. Initially, $50 \mu \mathrm{l}$ of umbilical cord blood was laced in $450 \mu \mathrm{l}$ of adult blood. Subsequently, a tenfold dilution factor was used, always transferring $50 \mathrm{ul}$ for each dilution step and resulting in blood samples containing fetal blood at dilutions of $1 / 10$, $1 / 100,1 / 1,000$, and $1 / 10,000$. This procedure resulted in 285 artificially diluted blood samples, containing from 1 to 1,000 fetal red blood cells per 10,000 adult red blood cells. In addition, undiluted blood samples of adults and umbilical cord were also evaluated as negative and positive controls, respectively. The samples were thoroughly homogenized during the dilution process and prior to analysis. Blood samples from puerperal women were used without any previous preparation.

2.3. Blood Sample Evaluations. The contents of fetal red blood cells and fetal hemoglobin in all blood samples (blood samples from male adults, umbilical cord, puerperal women, and serially diluted fetal blood samples) were evaluated by FCM and ABA, respectively. The FMH QuikQuant kit (IQ Products, Groningen, ME) was employed for the preparation of blood samples prior FCM based analysis, following the manufacturer's recommendations. Initially, serially diluted blood samples prepared as aforementioned and blood samples from puerperal women were diluted at 1/20 ratio with QuikQuant buffer. Then, $10 \mu$ l of diluted blood samples was fixed by addition of $0.75 \mathrm{ml}$ of glutaraldehyde $(0.04 \%$ in PBS; Sigma-Aldrich, Saint Louis, MO). During the period of fixation (10 minutes at room temperature), samples were intermittently vortexed. Thereafter, $1.5 \mathrm{ml}$ of Trillium IntraCell ${ }^{\mathrm{TM}}$ working solution was added to each samples tube, which remained incubated at room temperature for 10 minutes. Samples were washed by centrifugation and the supernatants were removed. Cell pellets were suspended with $10 \mu \mathrm{l}$ of Trillium FMH QuikQuant ${ }^{\mathrm{TM}}$ reagent, which contains fluorescein isothiocyanate-conjugated anti-HbF and propidium iodide, and $40 \mu \mathrm{l}$ QuikQuant ${ }^{\text {тм }}$ FMH Buffer working solution to prior incubation for 10 minutes in the dark. After the incubation period, samples were washed twice by centrifugation. Data acquisition was performed using a FACSVerse cytometer with FACSuite software (Becton Dickinson, San Jose, CA, USA) and a minimum of 50,000 cells were acquired. Data analysis was conducted using FlowJo software (Version 10; Tree Star, Ashland, OR, USA).

In parallel, the same blood samples were analyzed on an ABA ABL800 flex (Radiometer Medical, Brønshøj, Denmark). In this equipment, fetal hemoglobin is assessed by technics based on oximetry and spectrophotometry principles and does not require previous preparation of blood sample.

2.4. Statistics. The profile of the samples according to the variables under study was assessed by descriptive statistics. The accuracy of FCM and ABA to detect fetal blood laced with blood samples from male adults was further evaluated by plotting the receiver operating characteristic (ROC) curves for each of the methods. In order to analyze the relationship between the amounts of fetal blood detected by the two methods (FCM and ABA) the Spearman correlation coefficient was calculated. Because results included values equal to zero, all the values obtained by ABA and FCM were transformed as $\log _{10}(\mathrm{x}+0.5)$. Logarithmic values were also used for analyzing the agreement between the FCM and ABA by Bland-Altman method. The level of significance was set at $\mathrm{p}$ value $<0.05$. Statistical calculations were performed using Prism 6 (GaphPad Software; La Jolla, CA, USA) and the Statistical Analysis System (SAS) for Windows, version 9.2 (SAS Institute Inc., 2002-2008, Cary, NC, USA). 


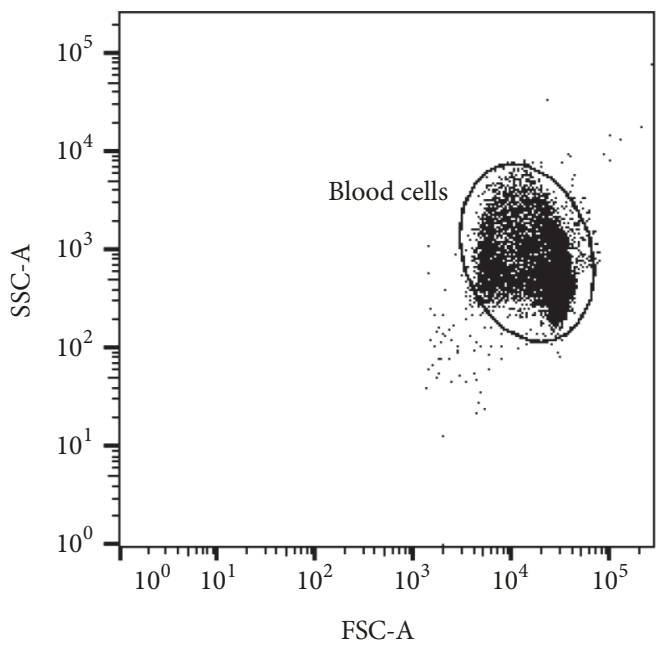

(a)

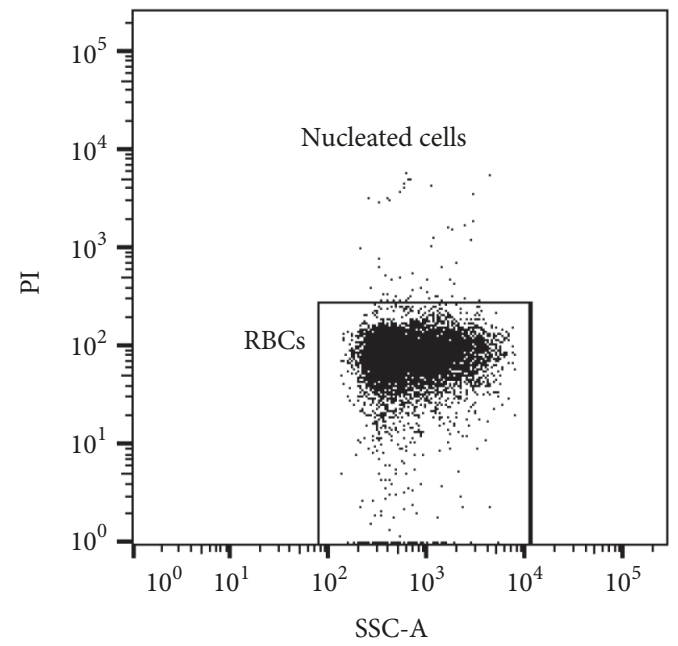

(b)

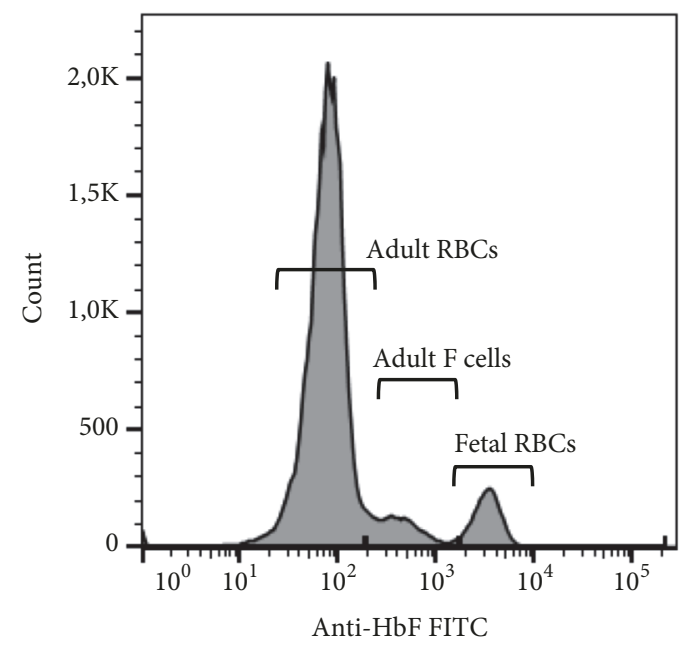

(c)

FIGURE 1: Flow cytometry-based analysis of blood samples, showing the gating strategy. The figure shows a representative sample at $1 / 10$ dilution of umbilical cord blood in blood of male adult. (a) Total blood cells were gated on logarithmic dot plot; (b) nucleated cells were excluded by gating the propidium iodide (PI) negative cell population. (c) Different red blood cell (RBC) populations were detected and quantified based on their fetal hemoglobin content. In addition to histogram, events within the gate of fetal RBCs were also evaluated by plotting the fluorescein isothiocyanate (FITC) channel against phycoerythrin (PE) and side-scatter (SSC) channels to confirm the percentage of events.

\section{Results}

3.1. Evaluation of Fetal Blood in Serially Diluted Blood Samples. The main characteristics of the subjects at the time of blood sampling were shown in Table 1, while Figure 1 depicts the gating strategy used in flow cytometry-based analysis to quantify fetal red blood cells in blood samples.

Both methods were able to differentiate between samples with and without fetal blood, while assessing blood samples of male adults containing fetal blood. However, this capacity was limited as dilution of fetal blood increased. Thus, ABA results were significantly different between samples with and without fetal blood up to dilution 1/10, while FCM results were significantly different up to dilution 1/100 (Supplementary Table 1).
The accuracy of FCM and ABA to detect fetal blood was evaluated by plotting the receiver operating characteristic (ROC) curves for each method (Figure 2 and Supplementary Figure 1). Overall, FCM (area under curve, AUC =0.8723) had better performance than ABA $(\mathrm{AUC}=0.6569)$ to detect the presence of fetal blood laced with blood of male adults (Figure 2). Additionally, ABA had lower specificity (59.65\%) and lower negative predictive value $(31.78 \%)$ compared with FCM $(78.95 \%$ and $58.44 \%$ respectively), indicating that assessment of FMH by ABA could be implied in a high probability of false positive results (Supplementary Table 2).

3.2. Correlation and Agreement between FCM and ABA. The correlation and agreement between results obtained through FCM and ABA were further compared following assessment 


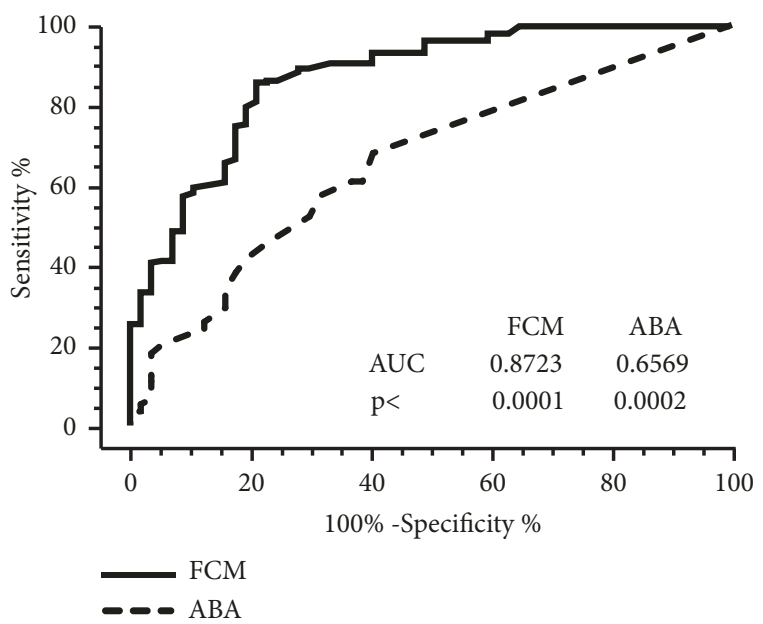

FIGURE 2: Receiver operating characteristic (ROC) curves comparing the performance of ABA and FCM in detecting fetal blood laced with blood from male adults. Blood samples were prepared by serially diluting umbilical cord $(\mathrm{n}=14)$ blood in blood of male adults $(n=57)$ at dilutions $1 / 10,1 / 100,1 / 1,000$, and $1 / 10,000$, resulting in 285 artificially diluted blood samples, containing from 1 to 1,000 fetal red blood cells per 10,000 adult red blood cells.

TABLE 1: Characteristics of the subjects at time of blood sampling.

\begin{tabular}{lcccc}
\hline & & Newborns & $\begin{array}{c}\text { Adult } \\
\text { males }\end{array}$ & $\begin{array}{c}\text { Puerperal } \\
\text { women }\end{array}$ \\
\hline Sex & Female & 8 & - & 40 \\
Gestational age & Male & 6 & 57 & - \\
(weeks) & & $36(33-41)$ & - & - \\
Age (years) & & - & $18-60$ & $18-42$ \\
& $\mathrm{~A}$ & 5 & 11 & 15 \\
ABO group & $\mathrm{B}$ & 3 & 3 & 4 \\
& $\mathrm{AB}$ & - & - & 1 \\
& $\mathrm{O}$ & 6 & 46 & 20 \\
RhD & Positive & 14 & 56 & 36 \\
& Negative & 0 & 4 & 4 \\
\hline
\end{tabular}

of both blood samples of male adults containing fetal blood at different dilutions (processed as aforementioned) and blood from puerperal women taken within 48 hours following delivery. Thus, low correlation (Figure 3(a)) and lack of agreement (Figure 3(b)) were found between results obtained by FCM and ABA for samples of male adults containing fetal blood. Similarly, there was no correlation (Figure 3(c)) or agreement (Figure 3(d)) between results obtained by FCM and $\mathrm{ABA}$ for the contents of fetal blood in samples from puerperal women.

FCM and ABA were compared for screening of FMH in puerperal women, taking into consideration thresholds (Supplementary Table 2) previously specified for these methods while assessing blood samples from male adults containing fetal blood. As indicated in Table 2, most of the puerperal women were categorized below the threshold specified for the lowest dilution of fetal blood. At 1/10 dilution; for example, $80 \%$ of the puerperal women (32 in 40 ) were below the thresholds established for FCM and ABA. However, as dilution increased, numbers of puerperal women ranged among the different classes and revealed a disparity between the two methods; i.e. ABA and FCM disagreed in indicating the same set of puerperal women as being above the thresholds. Thus, at $1 / 10,000$ dilution, $\mathrm{ABA}$ indicated $27.5 \%$ of the puerperal women (11 in 40) were above the thresholds while FCM indicated $17.5 \%$ (7 in 40), which comprised a different group of puerperal women (Table 2). Based on these results, it was determined that, at a critical level of $0.5 \%$, ABA indicated that $27.5 \%$ of puerperal women would have FMH while FCM did not detect FMH.

\section{Discussion}

In this study, $A B A$ was evaluated as a screening method for the detection of FMH. To our knowledge, this was the first time that such a method is systematically evaluated for this end. Additionally, accuracy of ABA to detect fetal blood was also evaluated and compared with FCM. Prevalence of isoimmunization due to $\mathrm{RhD}$ incompatibility between mother and her fetus has been lowered by the use of recommended prophylaxis [15]. However, FMH detection relies on methods with limited availability and, therefore, new automatized methods could benefit FMH diagnosis and treatment. Blood evaluation through $\mathrm{ABA}$ requires small amounts of blood and is less dependent on operator's practice and less expensive than FCM. Additionally, ABA is more accessible, since most of the intensive care units have this equipment [16].

Nevertheless, our results showed that evaluation of blood through ABA could be implied in overestimation of FMH. This feature is possibly a consequence of ABA's failure to differentiate between red blood cells from fetal blood and Fcells from adult blood. F-cells are red blood cells from adults containing $\mathrm{HbF}$. Although these cells can frequently be found in low amounts in the blood of healthy adults, their numbers are increased in certain inherited hemoglobin disorders or in acquired conditions, such as acute erythropoietic stress and cancer [17-21]. It was also reported that F-cells can be increased during pregnancy as a result of physiological response [22]. However, Pastoret et al. [23] established 2.2\% (0.3 to $11.3 \%)$ and $3.4 \%(0.9$ to $13.6 \%)$ as reference values of F-cells in pregnant and nonpregnant women, respectively. Similarly, we have found $2.6 \%$ (0.0 to $9.9 \%$ ) as reference value of F-cells in blood of male adult donors (data not shown).

Differently, detection of fetal red blood cells by FCM is based on the specific labeling of $\mathrm{HbF}$ by fluorophoreconjugated monoclonal antibodies (anti-HbF) [24, 25]. Additionally, anti-HbF specificity varies between $\mathrm{HbF}$ contained in fetal red blood cells and F-cells, favoring differentiation not only fetal red blood cells but also F-cells in blood of adults, as a consequence of differences in the resulting fluorescence intensity (Figure 1). Nevertheless, our data showed that anti$\mathrm{HbF}$ caused variable amounts of positive events that were detected within the gate of fetal red blood cells in blood 


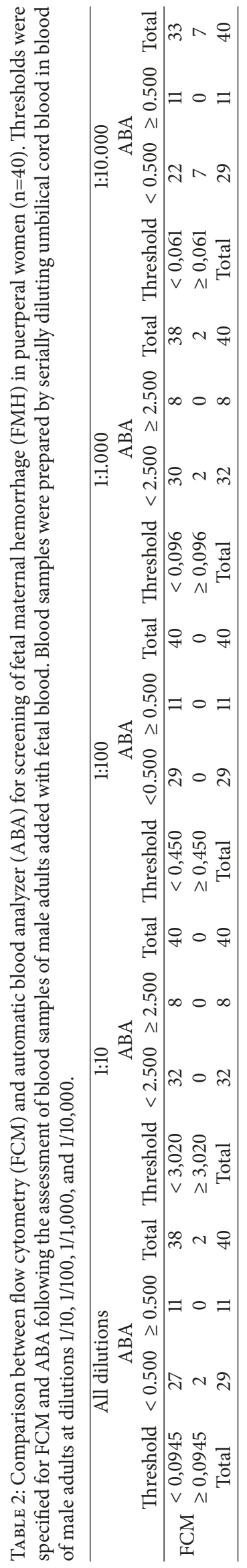




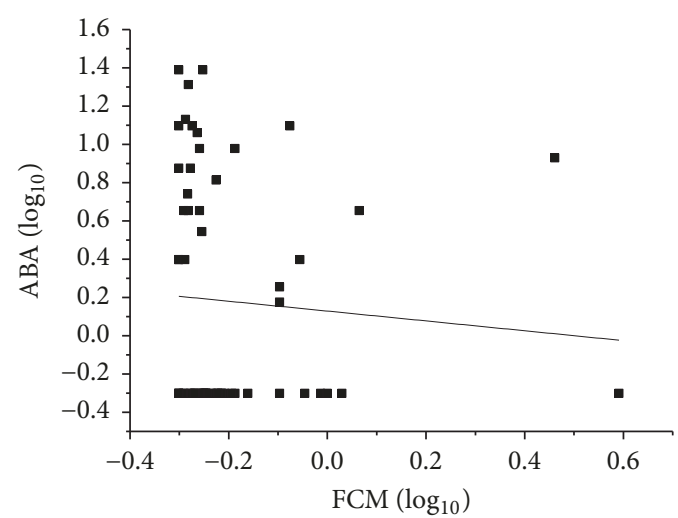

(a)

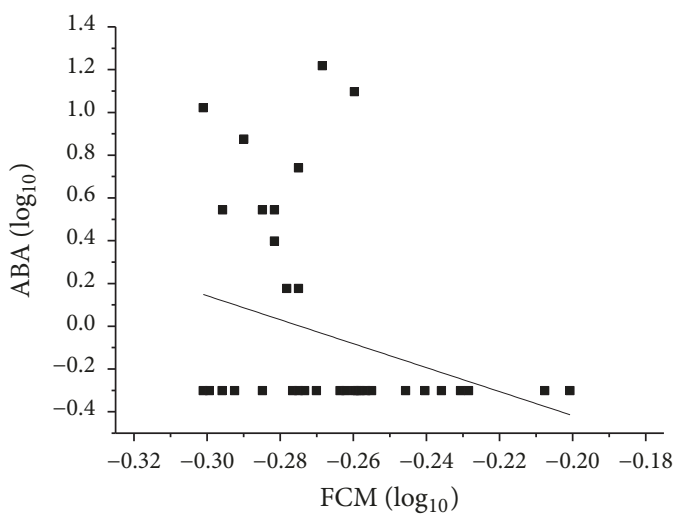

(c)

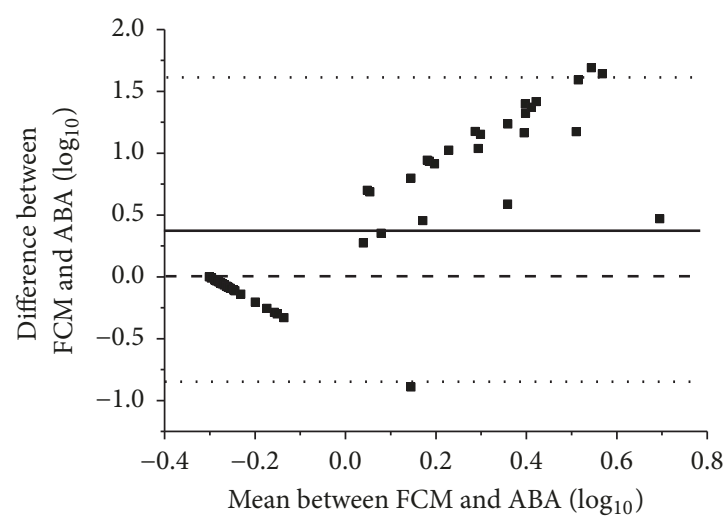

(b)

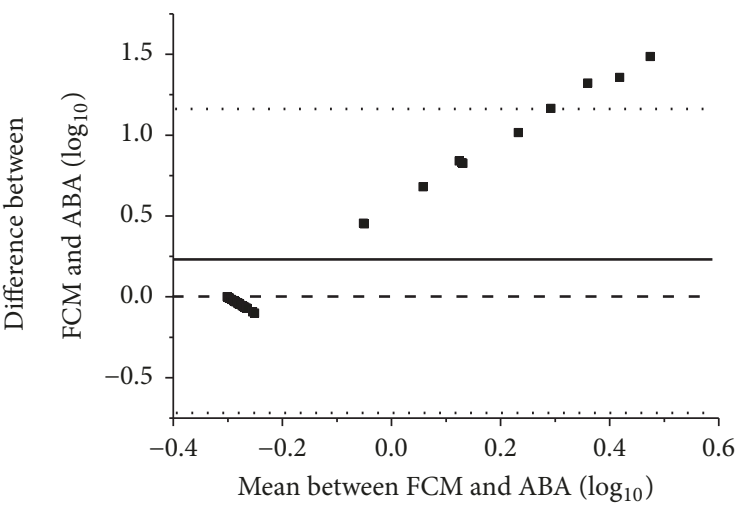

(d)

Figure 3: Parallel analysis of results obtained by FCM and ABA. (a) Low correlation (Spearman correlation: $r=-0.263, p=0.039$, and $n=57$ ) and (b) lack of agreement (bias $\mathrm{d}=0.342$, standard deviation $=0.6047$, and confidence interval $=95 \%[0.842 ; 1.526] \mathrm{p}=0.559$ ) were found for the results assessed in blood samples of male adults added with umbilical cord blood at dilutions 1/10, 1/100, 1/1,000, and 1/10,000. (c) No correlation (Spearman correlation: $\mathrm{r}=-0.316, \mathrm{p}=0.047, \mathrm{n}=40$ ) and $(\mathrm{d})$ agreement (bias $\mathrm{d}=0.233$, standard deviation $=0.481$, and confidence interval $=95 \%[-0.727 ; 0.397] \mathrm{p}=0.573$ ) were found for the results assessed in blood samples from puerperal women.

samples of male adults that were not added with umbilical cord blood. These positive events seem to be adult F-cells, at which the fluorescence intensity dragged them beyond the specific gate of adult F-cells. Although detected in low amounts, these positive events were responsible for certain loss of linearity of FCM, as dilution of fetal cells in blood samples of male adult increased (Supplementary Table 2).

FCM has been systematically evaluated for FMH diagnosis during the last decade and results have shown its advantages for assessment of fetal red blood cells and/or F-cells in blood samples $[20,22,23,26]$. Improved FCM protocols employing anti-HbF in combination with other monoclonal antibodies, such as anticarbonic anhydrase or anti-RhD, were also evaluated [7, 27]. However, volume of fetal blood leakage in $\mathrm{FMH}$ is rarely calculated in daily practice and, therefore, standard doses of anti-D immunoglobulin, ranging from 250 to $300 \mu \mathrm{g}$, are administered [26, 28, 29]. These doses are based on the recommendation to use $25 \mu \mathrm{g}$ of anti-D immunoglobulin for each $\mathrm{ml}$ of fetal blood leaked into the maternal blood compartment [30]. Without the accurate evaluation of FMH, this dose can represent an overtreatment.
However, with the use of this protocol, only 0.3 to $1.0 \%$ of treatment fails when the standard doses of immunoglobulin are employed on the $28^{\text {th }}$ week of gestation and within 72 hours postpartum [21, 31, 32].

Finally, although the predictive positive value specified for ABA (87.08\%, Supplementary Table 2) has initially suggested the ability of this method to detect fetal blood laced with blood from male adults, our results showed that ABA overestimates FMH and disagree with FCM on indicating puerperal women with FMH.

\section{Conclusion}

$\mathrm{ABA}$ is inadequate for being used to screen for or to measure FMH.

\section{Data Availability}

The data used to support the findings of this study are available from the corresponding author upon request. 


\section{Conflicts of Interest}

The authors declare that they have no conflicts of interest.

\section{Authors' Contributions}

Marcella R. Cardoso, Caroline N. de Souza-Araújo, and Maria Cecília R. Talarico performed inclusions of the subjects, blood sampling, experiments, and analyses of data. Marcella R. Cardoso, Fernando Guimarães, and Ricardo Barini performed analyses and interpretation of results and wrote the manuscript. Fernando Guimarães and Ricardo Barini designed the experiments. Juliana Heinrich-Mouçouçah is responsible for the automatic blood analyzer unit. Fernando Guimarães is responsible for the flow cytometry unit. Ricardo Barini was responsible for the research grant.

\section{Acknowledgments}

Authors are thankful to Helymar da Costa Machado for the support with statistical analysis. Authors are also thankful to William Barsé and Sylvio dos Santos Junior for the training on the use of ABL800 flex (Radiometer Medical, Brønshøj, Denmark). This study was supported by São Paulo Research Foudation (FAPESP, Grant no. 2016/05993-6) and Coordenação de Aperfeiçoamento Pessoal de Nível Superior (CAPES).

\section{Supplementary Materials}

Supplementary Table 1. Comparison between blood samples of male adults with and without fetal blood. All samples were assed for the amounts of fetal red blood cells or fetal hemoglobin by flow cytometry (FCM) or automatic blood analyzer (ABA), respectively. Blood samples were prepared by serially diluting umbilical cord blood in blood from male adult donors at dilutions 1/10, 1/100, 1/1,000, and 1/10,000. Supplementary Table 2. Comparison of flow cytometry (FCM) and automatic blood analyzer (ABA) at thresholds that maximizes sensitivity and specificity by combining all blood samples independently of dilution, and for each of the fetal blood dilutions. Blood samples were prepared by serially diluting umbilical cord blood in whole blood of male adult donors at dilutions 1/10, 1/100, 1/1,000, and 1/10,000. Supplementary Figure 1. Receiver operating characteristic (ROC) curves comparing the performance of ABA and FCM in detecting fetal blood laced with blood from male adults at dilutions 1/10 (A), 1/100 (B), 1/1,000 (C), and 1/10,000 (D). Samples were prepared by serially diluting umbilical cord blood in whole blood of male adult donors. AUC = area under curve. (Supplementary Materials)

\section{References}

[1] I. Hudecova and R. W. K. Chiu, "Non-invasive prenatal diagnosis of thalassemias using maternal plasma cell free DNA," Best Practice \& Research Clinical Obstetrics \& Gynaecology, vol. 39, pp. 1-11, 2016.
[2] A. B. Y. Cortey, "Prevention of fetomaternal rhesus-D alloimmunization. Practical aspects," Journal of Gynecology Obstetrics and Human Reproduction, vol. 35, pp. 1S123-1S130, 2006.

[3] A. R. Dis, "Non-host cells in the pathogenesis of autoimmune disease: a new paradigm?" Annals of The Rheumatic Diseases, pp. 518-520, 1999.

[4] B. J. Wylie and M. E. D’Alton, "Fetomaternal hemorrhage," Obstetrics \& Gynecology, vol. 115, no. 5, pp. 1039-1051, 2010.

[5] A. Pourazar, V. Homayouni, A. Rezaei, A. Andalib, and F. Oreizi, "The assessment of feto-maternal hemorrhage in an artificial model using anti-D and anti-fetal hemoglobin antibody by flow cytometry," Iranian Biomedical Journal, vol. 12, no. 1, pp. 43-48, 2008.

[6] E. Kleihauer and H. B. K. Braun, "Demonstration of fetal hemoglobin in erythrocytes of a blood smear," Klinische Wochenschrift, vol. 35, pp. 637-663, 1957.

[7] V. Porra, J. Bernaud, P. Gueret et al., "Identification and quantification of fetal red blood cells in maternal blood by a dual-color flow cytometric method: Evaluation of the Fetal Cell Count kit," Transfusion, vol. 47, no. 7, pp. 1281-1289, 2007.

[8] A. Ben-Haroush, A. Belkin, J. Chezar, J. Orlin, M. Hod, and J. Bar, "Comparison of two techniques for the evaluation of fetomaternal hemorrhage in RhD-negative women: Gel agglutination and haemoglobin F determination by flow cytometry," Acta Obstetricia et Gynecologica Scandinavica, vol. 86, no. 7, pp. 821-826, 2007.

[9] E. Chambers, L. Davies, S. Evans, J. Birchall, and B. Kumpel, "Comparison of haemoglobin F detection by the acid elution test, flow cytometry and high-performance liquid chromatography in maternal blood samples analysed for fetomaternal haemorrhage," Transfusion Medicine, vol. 22, no. 3, pp. 199-204, 2012.

[10] M. L. Kush, M. V. Muench, C. R. Harman, and A. A. Baschat, "Persistent fetal hemoglobin in maternal circulation complicating the diagnosis of fetomaternal hemorrhage," Obstetrics \& Gynecology, vol. 105, no. 4, pp. 872-874, 2005.

[11] M. P. Leers, H. M. Pelikan, T. H. Salemans, P. C. Giordano, and V. Scharnhorst, "Discriminating fetomaternal hemorrhage from maternal HbF-containing erythrocytes by dual-parameter flow cytometry," European Journal of Obstetrics \& Gynecology and Reproductive Biology, vol. 134, no. 1, pp. 127-129, 2007.

[12] H. De Wit, K. C. A. M. Nabbe, J. A. Kooren et al., "Reference values of fetal erythrocytes in maternal blood during pregnancy established using flow cytometry," American Journal of Clinical Pathology, vol. 136, no. 4, pp. 631-636, 2011.

[13] Radiometer Medical ApS: Manual de Referência do ABL800 FLEX 2004.

[14] L. Teitelbaum, A. Metcalfe, G. Clarke, J. S. Parboosingh, R. D. Wilson, and J. M. Johnson, "Costs and benefits of noninvasive fetal RhD determination," Ultrasound in Obstetrics \& Gynecology, vol. 45, no. 1, pp. 84-88, 2015.

[15] A. H. Chon, L. M. Korst, B. H. Grubbs, E. V. Kontopoulos, R. A. Quintero, and R. H. Chmait, "Risk factors for fetomaternal bleeding after laser therapy for twin-twin transfusion syndrome," Prenatal Diagnosis, vol. 37, no. 12, pp. 1232-1237, 2017.

[16] P. Oliver, A. Buno, R. Alvarez-Sala et al., "Clinical, operational and economic outcomes of point-of-care blood gas analysis in COPD patients," Clinical Biochemistry, vol. 48, no. 6, pp. 412418, 2015 .

[17] W. G. Wood, G. Stamatoyannopoulos, G. Lim, and P. E. Nute, "F cells in the adult: normal values and levels in individuals with 
hereditary and acquired elevations of Hb F,' Blood, vol. 46, no. 5, pp. 671-682, 1975.

[18] M. Wolk, J. E. Martin, and R. Constantin, "Blood cells with fetal haemoglobin (F-cells) detected by immunohistochemistry as indicators of solid tumours," Journal of Clinical Pathology, vol. 57, no. 7, pp. 740-745, 2004.

[19] M. Wolk, J. E. Martin, and C. Reinus, "Development of fetal haemoglobin-blood cells ( $\mathrm{F}$ cells) within colorectal tumour tissues," Journal of Clinical Pathology, vol. 59, no. 6, pp. 598-602, 2006.

[20] K. Y. Italia, R. Colah, and D. Mohanty, "Evaluation of F cells in sickle cell disorders by flow cytometry - comparison with the Kleihauer-Betke's slide method," International Journal of Laboratory Hematology, vol. 29, no. 6, pp. 409-414, 2007.

[21] A. Yu, E. Morris, R. Adams, and M. K. Fung, "Obstetrics and gynecology physician knowledge of Rh immune globulin prophylaxis," Transfusion, vol. 57, no. 6, pp. 1385-1390, 2017.

[22] E. A. Fong, J. Finlayson, F. Robins et al., "Evaluation of a new rapid anti-HbF FITC assay, Trillium QuikQuant, for detection and quantitation of foetomaternal haemorrhage," International Journal of Laboratory Hematology, vol. 35, no. 1, pp. 106-110, 2013.

[23] C. Pastoret, J. L. Priol, T. Fest, and M. Roussel, "Evaluation of FMH QuikQuant for the detection and quantification of fetomaternal hemorrhage.," Cytometry. Part B, Clinical cytometry, vol. 84, no. 1, pp. 37-43, 2013.

[24] B. H. Davis, S. Olsen, N. C. Bigelow, and J. C. Chen, "Detection of fetal red cells in fetomaternal hemorrhage using a fetal hemoglobin monoclonal antibody by flow cytometry," Transfusion, vol. 38, no. 8, pp. 749-756, 1998.

[25] M. G. Farias, S. Dal Bó, S. M. D. Castro et al., "Flow Cytometry in Detection of Fetal Red Blood Cells and Maternal F Cells to Identify Fetomaternal Hemorrhage," Fetal and Pediatric Pathology, vol. 35, no. 6, pp. 385-391, 2016.

[26] A. Gielezynska, A. Stachurska, J. Fabijanska-Mitek, M. Debska, K. Muzyka, and E. Kraszewska, "Quantitative fetomaternal hemorrhage assessment with the use of five laboratory tests," International Journal of Laboratory Hematology, vol. 38, no. 4, pp. 419-425, 2016.

[27] S. A. Akbar and P. R. Brown, "Measurement of human erythrocyte CAI and CAII in adult, newborn, and fetal blood," Clinical Biochemistry, vol. 29, no. 2, pp. 157-164, 1996.

[28] H. Qureshi, E. Massey, D. Kirwan et al., "BCSH guideline for the use of anti-D immunoglobulin for the prevention of haemolytic disease of the fetus and newborn," Transfusion Medicine, vol. 24, no. 1, pp. 8-20, 2014.

[29] F. B. Clausen, M. Christiansen, R. Steffensen et al., "Report of the first nationally implemented clinical routine screening for fetal RHD in D-pregnant women to ascertain the requirement for antenatal RhD prophylaxis," Transfusion, vol. 52, no. 4, pp. 752-758, 2012.

[30] H. G. A. D. Klein, "Haemolytic disease of the fetus and newborn," in Mollison's Blood Transfusion in Clinical Medicine, pp. 522-527, 12th edition, 2014.

[31] I. M. Bromilow and J. K. Duguid, "Measurement of fetomaternal haemorrhage: a comparative study of three Kleihauer techniques and tow flow cytometry methods," Clinical \& Laboratory Haematology, vol. 19, pp. 137-142, 1997.

[32] N. Ochsenbein-Imhof, A. F. Ochsenbein, B. Seifert, A. Huch, R. Huch, and R. Zimmermann, "Quantification of fetomaternal hemorrhage by fluorescence microscopy is equivalent to flow cytometry," Transfusion, vol. 42, no. 7, pp. 947-953, 2002. 


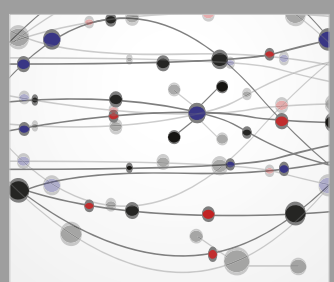

The Scientific World Journal
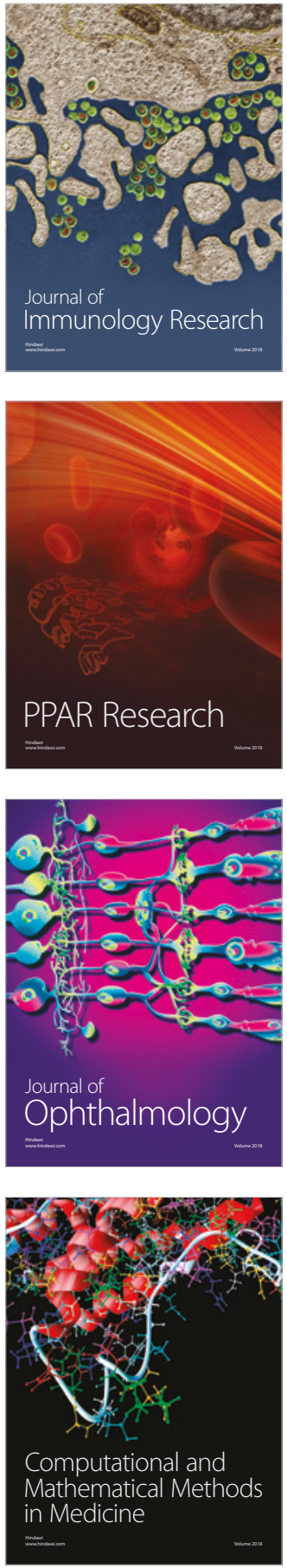

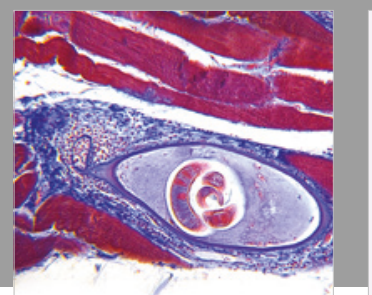

Gastroenterology Research and Practice

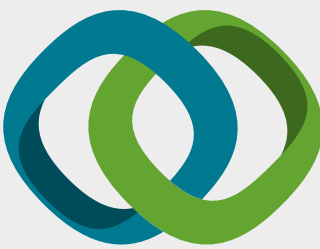

\section{Hindawi}

Submit your manuscripts at

www.hindawi.com
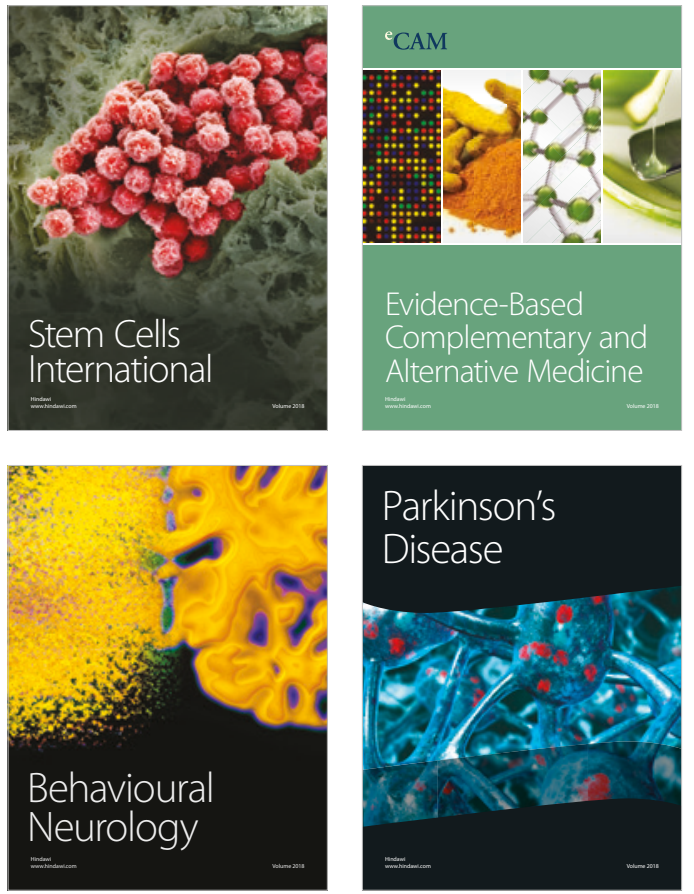

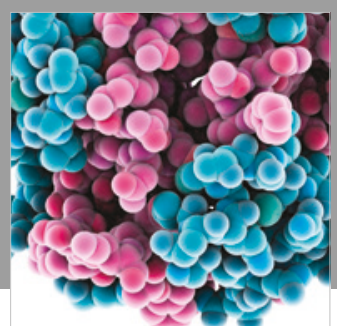

ournal of

Diabetes Research

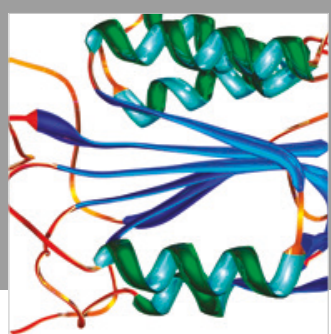

Disease Markers
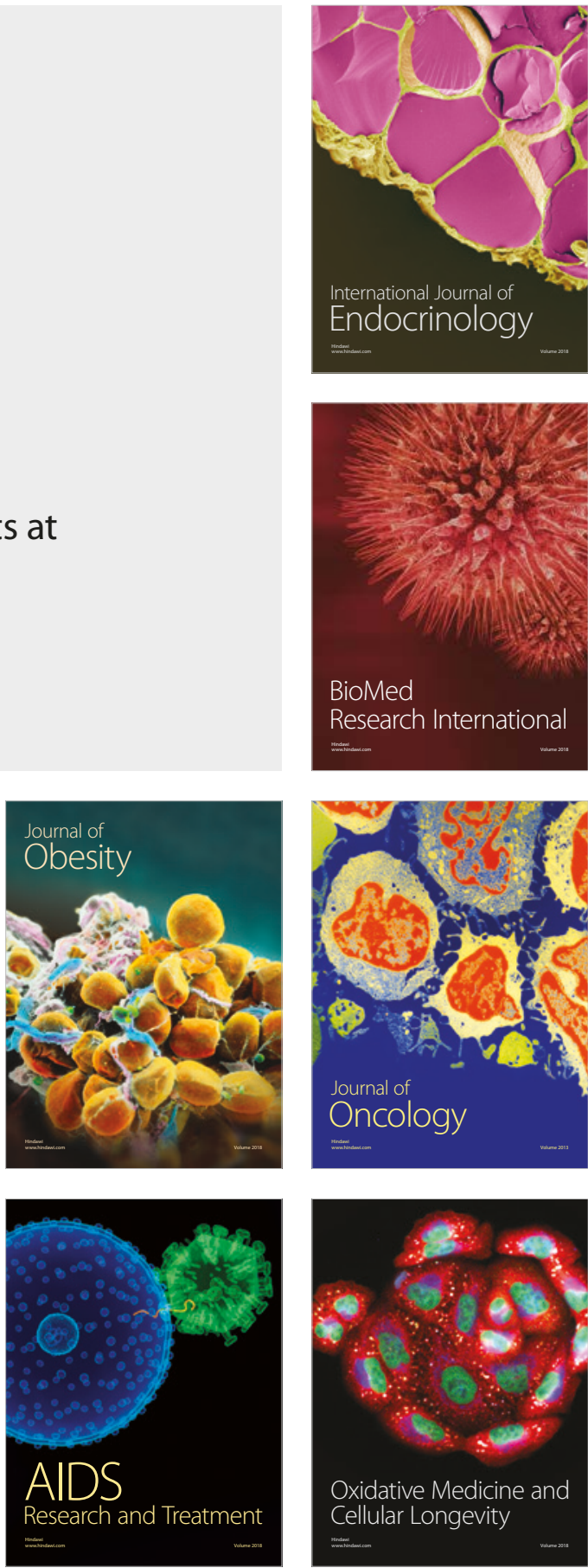